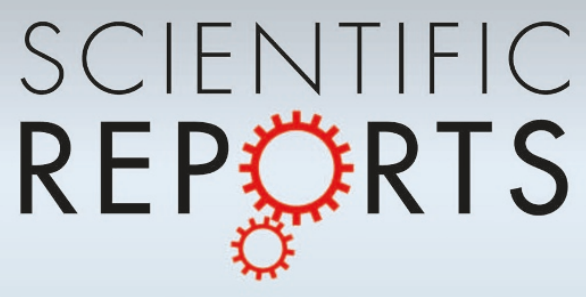

OPEN

SUBJECT AREAS:

POLYMERS

NANOPARTICLES

STRUCTURAL PROPERTIES

Received

7 August 2014

Accepted

16 September 2014

Published

15 October 2014

Correspondence and requests for materials should be addressed to S.J.L. (sjlee@postech. ac.kr)

\section{Nanoparticle role on the repeatability of stimuli-responsive nanocomposites}

\author{
Sungsook Ahn ${ }^{1,2} \&$ Sang Joon Lee ${ }^{1,2}$
}

\begin{abstract}
'Biofluid and Biomimic Research Center, Pohang University of Science and Technology, Pohang, 790-784, Korea, ${ }^{2}$ Department of Mechanical Engineering, Pohang University of Science and Technology, Pohang, 790-784, Korea.
\end{abstract}

Repeatability of the responsiveness with time is one important concern for effective durable functions of stimuli-responsive materials. Although the increase in the yield and tensile strength of the hybrid composite materials by nanoparticle (NP) incorporation has been reported, exact NP effect on stimuli-responsiveness is rarely reported. In this study, a set of nanoscale actuating system is demonstrated by a thermo-sensitive process operated by polyethylene glycol (PEG) linked by gold nanoparticle (AuNP). This designed nanocomposite exclusively provides an artificial on/off gate function for selective passages of permeate molecules. The results demonstrate high repetition efficiency with sharp responding in a timely manner. In terms of the morphology changes induced by repeated swelling-deswelling mechanics, the nanocomposite exhibits phase separation between AuNP clusters and PEG domains. This leads to a delay in responsiveness in a cumulative way with time. Acting as stable junction points in the nanocomposite network structures, the incorporated AuNPs contribute to maintain repeatability in responsiveness. This study contributes to new-concept smart material design and fundamental understanding on the hybrid nanomaterials for various applications in terms of a dynamic mechanical behavior.

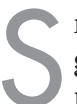

mart or stimuli-responsiveness ${ }^{1}$ that modifies properties in response to external stimuli represents a growing cadre of materials that support various applications, including controlled-release agents ${ }^{2-3}$, responsive coatings ${ }^{4}$, and adaptive shape-memory materials ${ }^{5}$. In addition to the separation of molecules by size and charge for which traditional membranes are employed, responsive membranes such as human skin demonstrate a smart valve function controlled by external stimuli ${ }^{6}$. Recent advances in nanotechnology have been interested in designing smart materials that mimic the processes found in natural systems. In this point, synthetic membranes capable of responsive functions find broad ranges of applications.

Different from homogeneous polymeric materials, nanocomposites composed of metal nanoparticles (NPs) dispersed in a polymer matrix exhibit novel properties ${ }^{7}$. Composed into a responsive composite material, the role of each component is cooperative leading to characteristic collective property. In this point, blends and chemically-linked particle-polymer systems are physically different in many aspects. Composite materials are widely reported to upgrade stiffness and strength of a polymeric materials without a significant loss in resilience or toughness. One example, adding mica to nylon produces a five-fold increase in the yield and tensile strength of the material ${ }^{8-9}$. Even though precise control of NP dispersion in the polymeric matrix has been focused, this is typically hurdled by thermodynamic instability and easy agglomeration as a result of high surface free energy of NPs. Recent advances exploit both the enthalpic and entropic interactions that direct the spatial distribution of NPs in a polymeric matrix to enhance macroscopic performance of materials. Even though versatile noncovalent methods have been employed to assemble individual NPs into designed structures ${ }^{10-12}$, NPs have been covalently collected for increased stability and uniformity. Once covalently linked, NP-embedded nanocomposite generates unique physical properties contributed by collective interactions of individual NPs ${ }^{13}$.

The responsive mechanism of stimuli-responsive materials depends on many factors such as conformational changes and phase segregation of the employed polymers as well as polymer-water/polymer-polymer interaction in the practical temperature ranges ${ }^{13-19}$. In terms of responsiveness, the properties of the composite materials are complicated due to compositional complexity. Recent advances exploit both enthalpic and entropic interactions to direct the spatial distribution of NPs and thereby control the macroscopic performance of the material. NPs influence the phase-separation kinetics and significantly alter the coarsening dynamics of polymer mixtures ${ }^{20}$. Theoretical studies of NP-filled mixtures suggest the existence of distinct pattern formation at early stages of phase separation ${ }^{21-22}$ and a subsequent slowing of domain growth at later times ${ }^{23}$. Experiments have confirmed a substantial slowing of phase separation by NP addition to a polymer mixture ${ }^{24-25}$. In this study, the role of NPs in a stimuli-responsiveness of nanocomposites is investigated in terms of stable repetition of the mechanistic swelling- 
deswelling mechanistic cycles. The role of NPs in the composite materials are investigated in terms of the dynamical structural change and durability in mechanical responsiveness.

\section{Results}

Citrate-stabilized colloidal AuNP (naked AuNP) of $20 \mathrm{~nm}$ average diameter are prepared ${ }^{26,27}$ in aqueous standard solution adjusted to around $2.4 \times 10^{12}$ AuNPs/mL (Supporting Information). Binary and quaternary thiol end-capped functional polyethylene glycol (PEG) are incorporated for AuNP interconnection (Figure 1a) ${ }^{10-11}$. Molecular weight between the junction point $\left(\mathrm{M}_{\mathrm{p}}\right)$ is controlled by their characteristic structures: 2 PEG 3400 has $\mathrm{M}_{\mathrm{p}}=3400\left((\mathrm{EO})_{\mathrm{n}}\right.$, $\mathrm{n}=77)$ and 2PEG 10000 has $\mathrm{M}_{\mathrm{p}}=10000\left((\mathrm{EO})_{\mathrm{n}}, \mathrm{n}=227\right)$ for binary-functional PEGs. Meanwhile, 4PEG 10000 has $M_{p}=10000$ $\left((E O)_{n}, n=227\right)$ and 4 PEG 20000 has $M_{p}=20000\left((E O)_{n}, n=454\right)$ for quaternary-functional PEGs. In addition, the number of incorporated PEG molecules is controlled to $\times 10, \times 50$, and $\times 100$ times of the AuNPs for each system, considering multi-reactive sites on a single AuNP (Supporting Information). Network is hardly formed if binary PEG monomers are only activated. However, interconnected composite clusters are formed as a result of multiple reactivity of AuNP surface to thiol end groups of the PEGs. NPs are typically considered to perform a random walk on the lattice model (Figure $\mathrm{S} 1)^{28}$. Effective particle mobility on the lattice is controlled by specific particle-to-solvent step ratios ${ }^{29}$. Considering that each lattice contains one AuNP, and these lattices are diversely interconnected according to the molecular weight and the structure of the PEGs. Multiple PEG linkages are attached to the AuNP surface to form networked assemblies. Mono-tethered AuNPs are analogous to single-tailed surfactants or diblock copolymers, whereas multi-tethered AuNPs work as junction points in the network. They also possess additional levels of complexity and anisotropy that can be exploited in self-assembly. The crosslink density $(\rho)$ of the fully-linked network is inversely proportional to the molecular weight between the junction points $\left(\mathrm{M}_{\mathrm{p}}\right)$.

Figure $1 \mathrm{~b}$ presents small-angle X-ray scattering (SAXS) results conducted at the $4 \mathrm{C}$ beam line of the Pohang Accelerator Laboratory (Pohang, Korea) with the designed AuNP-PEG nanocomposite networks in aqueous solution. Aqueous PEG solutions exhibit unique physical property depending on the molecular weight: the solubility decrease and/or phase separation occurs above the critical temperature (lower critical solution temperature, LCST) 2-3,30-31. $^{2}$ Different from typical responsive materials exhibiting isotropic changes in their scales, the organic-inorganic hybrid composites display deviation from the typical rubber elasticity by characteristic contribution of individual components. The nanocomposites designed in this study display dual regions for responsiveness: a large-size domain is fixed while a small-scale domain is responsive to an environmental stimuli. At a fixed PEG concentration $(\times 100)$, temperature-responsiveness of PEGs are compared at three different temperature conditions $\left(20,40\right.$ and $60{ }^{\circ} \mathrm{C}$ ) (Figure $\left.1 \mathrm{~b}\right)$. For all the systems there is a characteristic $q$ value $\left(q^{*}\right)$ from which the spectrum is diverged responding to each temperature condition: $q^{*}=$ $0.7 \mathrm{~nm}^{-1}, 0.4 \mathrm{~nm}^{-1}, 0.4 \mathrm{~nm}^{-1}$ and $0.6 \mathrm{~nm}^{-1}$ for 2PEG 3400, 2PEG 10000, and 4PEG 10000 and 4PEG 20000, respectively. From those $q^{*}$, the solubility of the composite network decreases according to the temperature increase from 20 to $60{ }^{\circ} \mathrm{C}$, exhibiting characteristic LCST, except lowest molecular weight 2PEG 3400 system $^{30-31}$. This indicates that the designed AuNP-PEG composites consist of dual domains: non-responsive stable domain in large-scale (low $q$ region) and stimuli-responsive flexible domain in small-scale (high $q$ region). However, size-definable structures are generated only for 2PEG 10000 and 4PEG 10000 system marked at $q_{1}, q_{2}$, and $q_{3}$, for each temperature condition. This moves to lower $q$ region with temperature increase, exhibiting larger size formation at increased temperature. With the increase in the incorporated PEG amount, the $q^{*}$ point moves to the higher $q$ region, indicating smaller size scale, from which the materials become responsive (Figure S2). We suggest this dual regions come from the characteristic hardness of the embedded metal nanoparticle and also simultaneously responsive polymeric matrix, and this combined properties are unique in composite materials.

We obtained same batch of the nanocomposites and after purification we obtained characteristic physical properties (SAXS, UV-vis, TEM, XNI, XMI) of the designed nanocomposites and used them for all the mass transport experiments. Temperature-responsive nano-scale networks are illustrated in Figure 1c. The designed nanocomposites are embedded in poly(vinyl alcohol) (PVA) gel matrix (3 wt $\%, \mathrm{M}_{\mathrm{w}} \sim 72,000 \mathrm{Merck}$ ), keeping the temperature-responsiveness of nanocomposites without leakage and shape deformation, significant swelling or shrinking in the designed experimental conditions $^{32}$. Even at increased temperature several times above and below the transition point, AuNP-PEG composites released from the PVA matrix are not detected. The AuNP-PEG nanocomposite embedded in PVA matrix exhibits differentiated paths for the selected permeates according to the temperature condition. Structural transition occurs by triggered temperature depending on the nanocomposite structures. The correlation length $(\xi)$, the distance between the junction points of the network, changes according to the temperature: compared with the condition of lower temperature of highly swollen state [I], the $\xi$ values become larger by the shrinkage at an increased temperature [II]. According to the temperature change, the structure of the designed nanocomposite is effectively controlled for the transport of permeate molecules. With increased temperature, the selected permeates pass the nanocomposites more effectively through larger $\xi$ [II] than through smaller $\xi$ at lower temperature [I].

Mass transport is controlled in a designed diffusion cell in which selected permeate molecules (Rhodamine 6G or Pyrene) ${ }^{33-34}$ pass through the designed nanocomposite. The AuNP-PEG nanocomposite embedded in PVA matrix is loaded in the humidity- and temperature-controlled glass tube of $0.1 \mathrm{~cm}$ diameter and $5 \mathrm{~cm}$ length and connected to the syringe pump (Figure 2a). The retention time $\left(t_{n}\right)$ of the permeates is recorded by direct observation of the eluted solution color or fluorescence signal in continuous batch (Supporting Information). Relatively sharp point of $\mathrm{t}_{\mathrm{n}}$ is determined by the time from which the presence of the permeate at a detectable concentration is verified (Figure $2 b$ ). For all the designed systems, there is a sharp increase in the signal determined as a retention time $\left(t_{n}\right)$. To determine $t_{n}$ for each nanocomposite switching on/off temperature-sensitive valve, nanocomposite packing geometry and flow rate are carefully controlled at two temperature conditions (20 and $60^{\circ} \mathrm{C}$ ) switched in a minute interval. The controlled low rate of $0.001 \mu \mathrm{L} / \mathrm{min}$ through $2 \mathrm{PEG} 10000$ nanocomposite, proper repetition signals of Rhodamine $6 \mathrm{G}$ are obtained in a timely manner as illustrated in Figure $2 \mathrm{c}$. The temperature changes are recorded in a minute interval marked by solid black lines, and sharp increase in the signals of permeate molecules are exclusively detected during the condition of an elevated temperature.

The synchronized signals are displayed in Figure 2d, reflecting the repeatability of the temperature-responding valve function of the designed nanocomposites. The on/off switching is controlled regularly by detected permeate molecules of a fixed concentration. The temperature change is recorded with a minute interval (black line) and the detected $t_{n}$ of permeate molecule (dotted red line) is controlled accordingly. The time-dependent swelling-deswelling cycling repetition is compared for 2PEG 10000 and 4PEG 10000 systems. At an initial stage, the repetition cycle is regular for both systems. 2PEG 10000 system shows unchanged repetition pattern during the measurement time duration. However, 4PEG 10000 system exhibits delayed responsiveness between the temperature onset and permeate elution response as time goes on, which is quantified by the length of the arrows. 


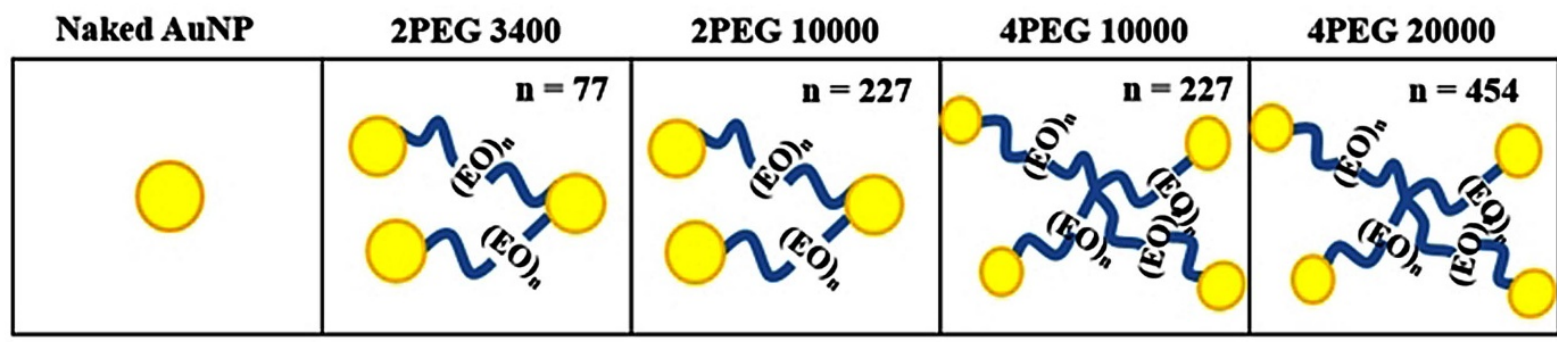

b
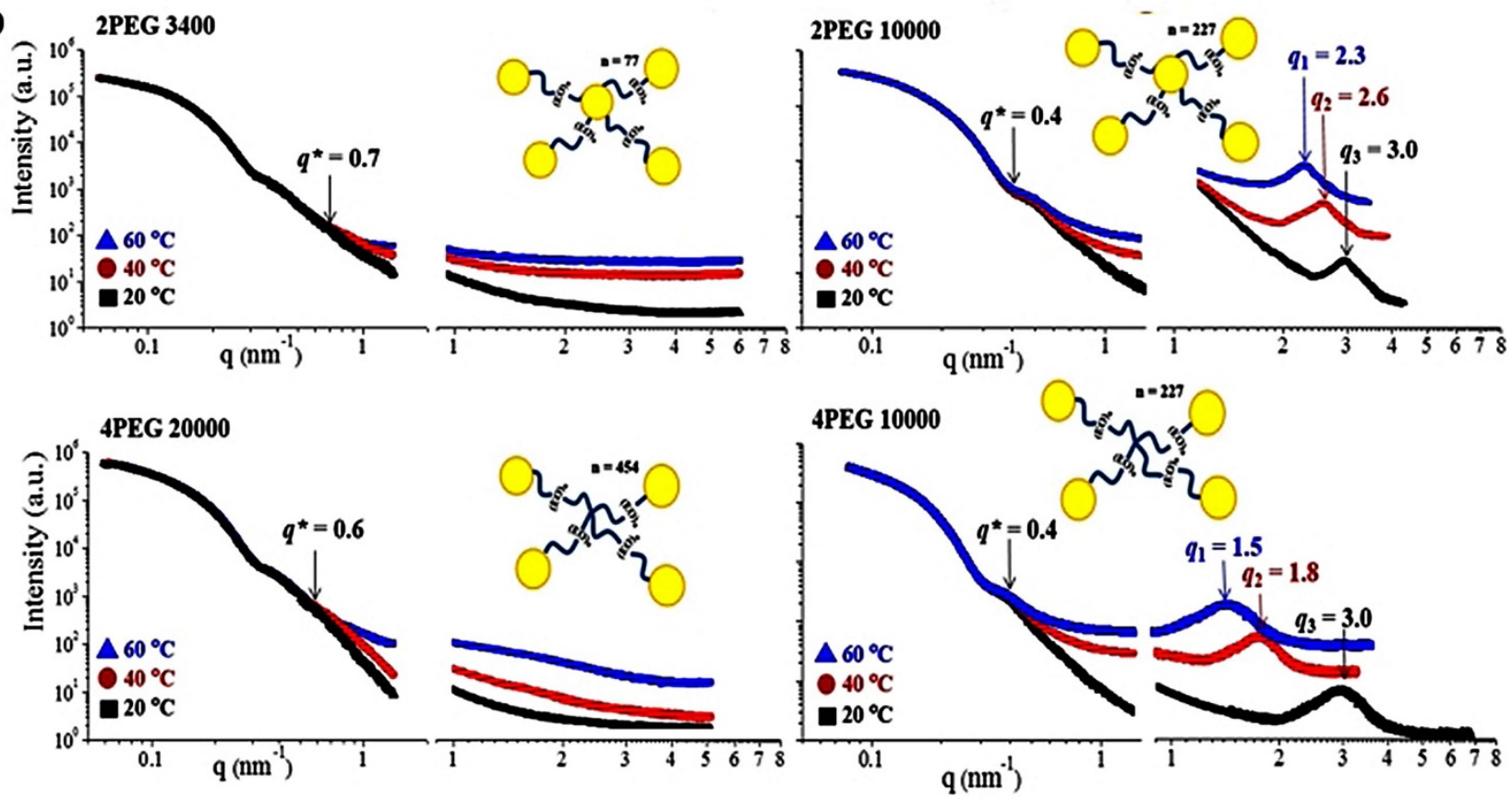

C
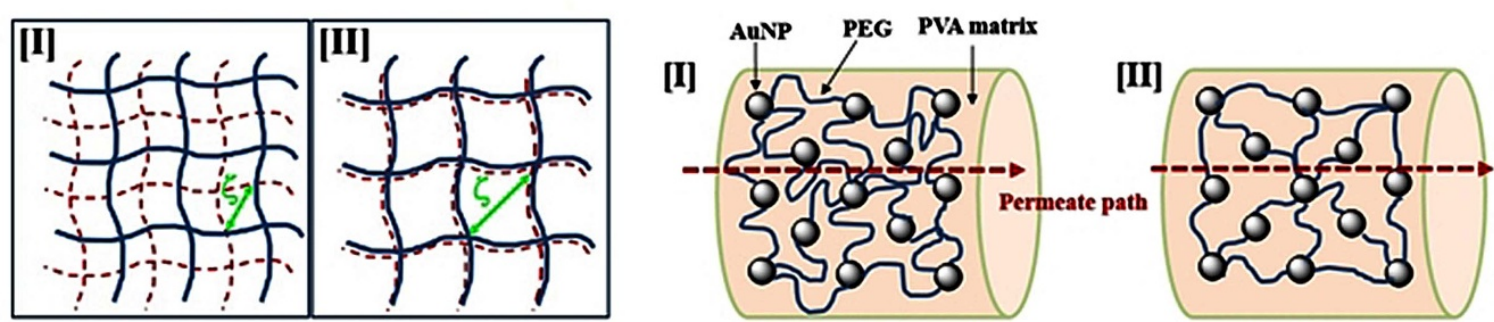

Figure $1 \mid$ (a) Gold nanoparticle (AuNP) and structure of the functional polyethylene glycol (PEG) for interlinking. Due to multi reactive sites of the AuNP to thiol group of PEGs various networked structures are generated. Binary functional PEGs having number of EO unit as of $\mathrm{n}=77$ is $2 \mathrm{PEG} 3400$ and $n=227$ is 2PEG 10000. Quaternary functional PEGs having EO unit as of $n=227$ is 4 PEG 10000 and $n=454$ is 4PEG 20000. (b) Small angle X-ray scattering (SAXS) results of the designed AuNP-PEG nanocomposites in solution state in broad $q$ ranges (two SDD distance conditions are combined) for 2PEG 3400, 2PEG 10000, 4PEG 10000 and 4PEG 20000 linked AuNP clusters. There is a critical $q$ value (marked by $q^{*}$ ) from which temperatureresponsiveness is diversified indicating dual regions of the designed network: stable large scale domain and responsive small scale domain. In two systems of 2PEG 10000 and 4PEG 10000, definable size is determined according to the temperature. (c) Illustration of the dual-responsive network structures. Bold lines denote stable connection, while dotted lines indicates flexibly responsive chains by the external stimuli (left). Pore size variation of the nanocomposites embedded in PVA matrix induced by the stimuli-responsive PEGs through which permeates are transported. Swollen PEGs generates smaller path for permeate molecules [I], while shrunken PEGs allow relatively wide pathway for effective transport of permeates [II].

The structures of the employed AuNP-2PEG 10000 and AuNP-4PEG 10000 clusters are compared before and after the cycling repetitions (Figure $3 \mathrm{a}$ ). The measurement temperature is controlled as $20{ }^{\circ} \mathrm{C}$. At the first line of the transmission electron microscopy (TEM) images, the AuNPs in the composite clusters are effectively contrasted by high electron density against the linked organic PEGs. A representative image of the citrate-covered spherical AuNPs without ligand linkage (naked AuNPs) verifies $20 \mathrm{~nm}$ average diameter of a single AuNP. Before the cycling repetition, the AuNPs connected by binary 2PEG 10000 show rather more closely packed clusters compared with those con- nected by quaternary 4PEG 10000 of longer interparticle distances. Even after the cycling of continuous valve work until around $500 \mathrm{~min}$, the AuNP clusters connected by 2PEG 10000 have no clear difference. Meanwhile, 4PEG 10000 exhibits prominently distinguishable changes which become highly aggregated AuNP clusters in TEM images. The structural difference in nanoscale composite cluster is dominated by $\mathrm{M}_{\mathrm{p}}$ as well as the structure of the linker-molecules. By the quaternary structure, 4PEG 10000 actually has $M_{P}$ value of 20000 crossed at the center, this induces polymer-dominated phase behavior against the AuNP-linked hybrid structures. 
a

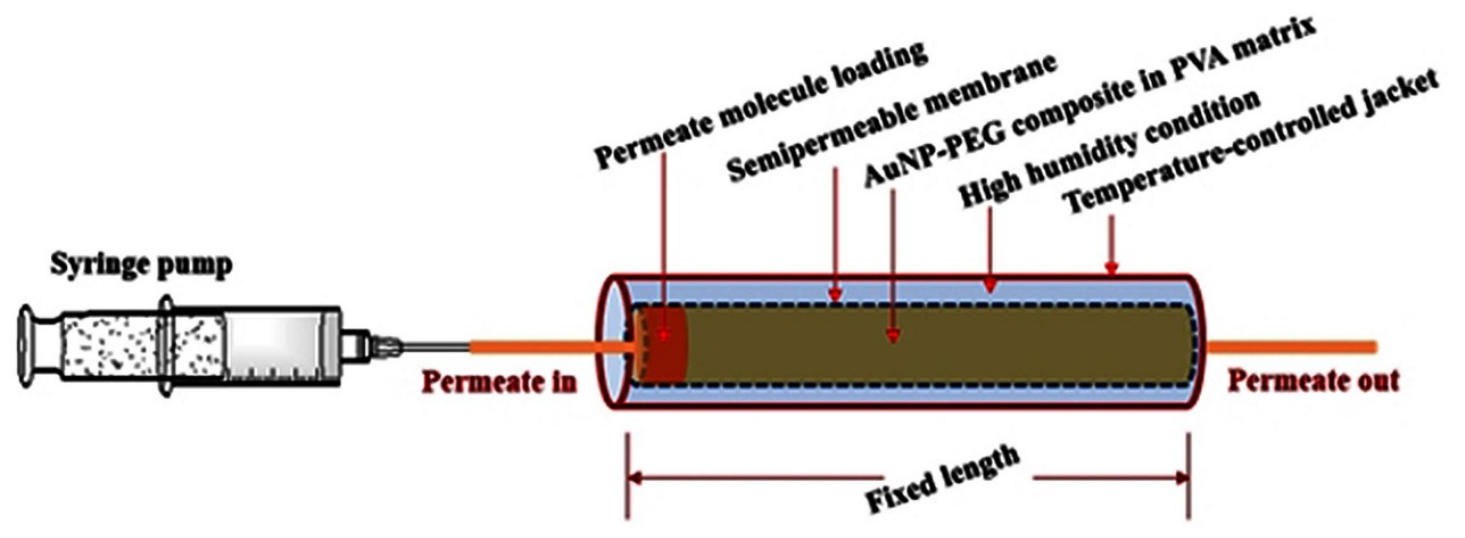

b
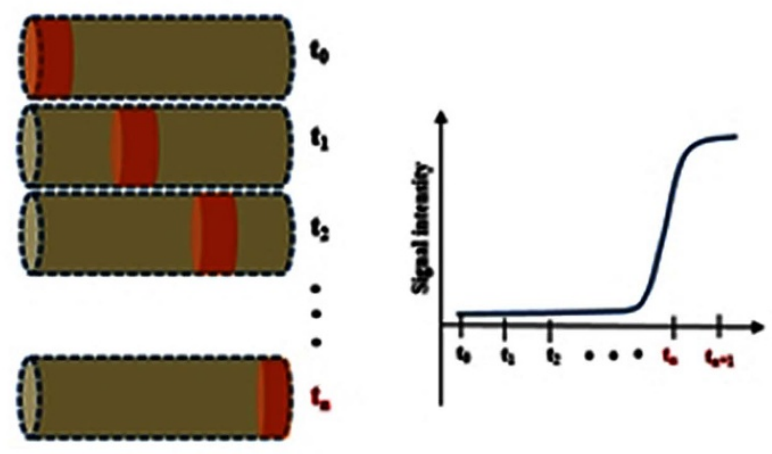

c

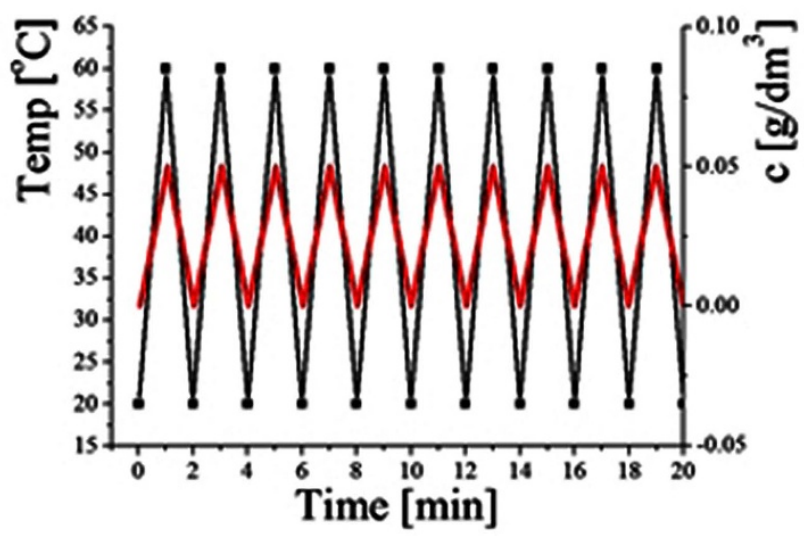

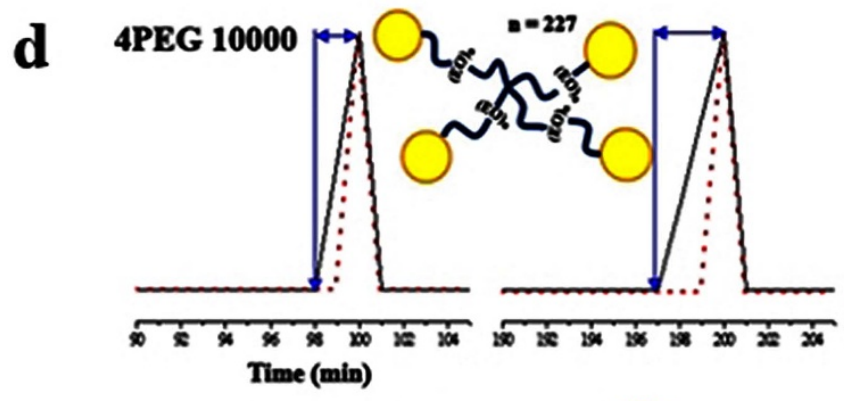
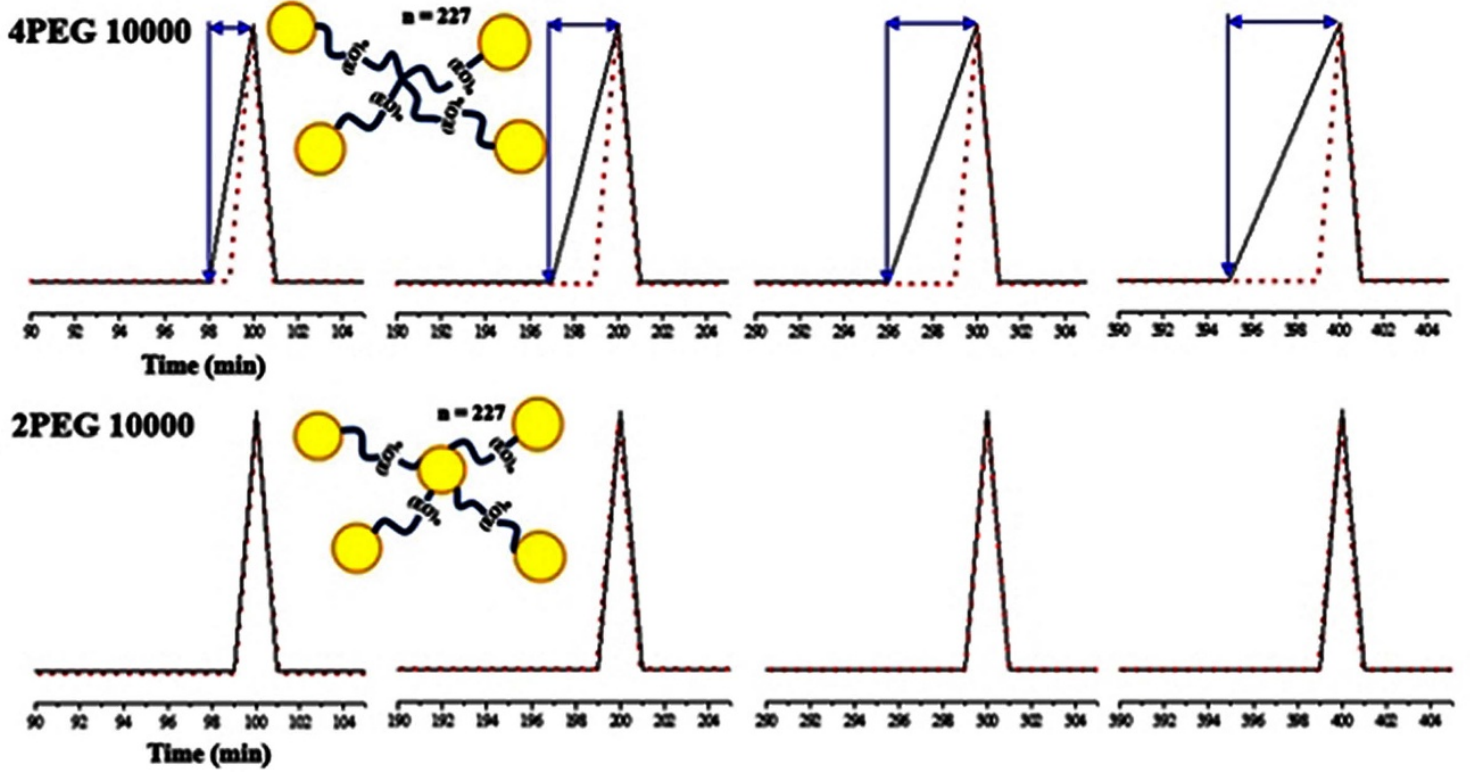

Figure 2 (a) Experimental set-up for mass-transport. Permeate molecules are loaded at the entrance of the diffusion cell and flow rate are carefully controlled. (b) Retention time ( $\left.t_{n}\right)$ of each system is determined from which the permeate molecules are detected at the outlet flow. (c) Repetition cycles of the temperature triggering to the nanocomposite-loaded diffusion cell and molecular responding of permeate detection. The results are obtained by Rhodamine 6G passing through 2PEG 10000 nanocomposites and recorded in a minute interval. (d) Time-dependent repetition cycles for two nanocomposite systems of 2PEG 10000 and 4PEG 10000. Temperature triggering and permeate detection occur simultaneously for 2PEG 1000 system, while there is cumulative delay for 4PEG 10000 system. All the figures were created by the authors.

In the second line of Figure 3a, X-ray nano imaging (XNI) results are displayed. The images are obtained at the $7 \mathrm{C}$ beam line in $\mathrm{PAL}$ (Supporting Information). Beam size is adjusted in $100 \mu \mathrm{m} \times$ $100 \mu \mathrm{m}$ at $7 \mathrm{keV}$ energy. Spatial resolution is approximately $200 \mathrm{~nm}$ and field-of-view (FOV) is adjusted to $20 \mu \mathrm{m} \times 20 \mu \mathrm{m}$ (the images are put together to get a whole image). Before cycling, both type of AuNP clusters exhibit homogeneous small dots. After the repetition cycling, 2PEG 10000 linked system does not generate prominent difference. Meanwhile, dotted patterns of 4PEG 10000 linked clusters become prominently larger after the cycled repetition by induced phase separation of AuNPs from the PEG polymer domain. 

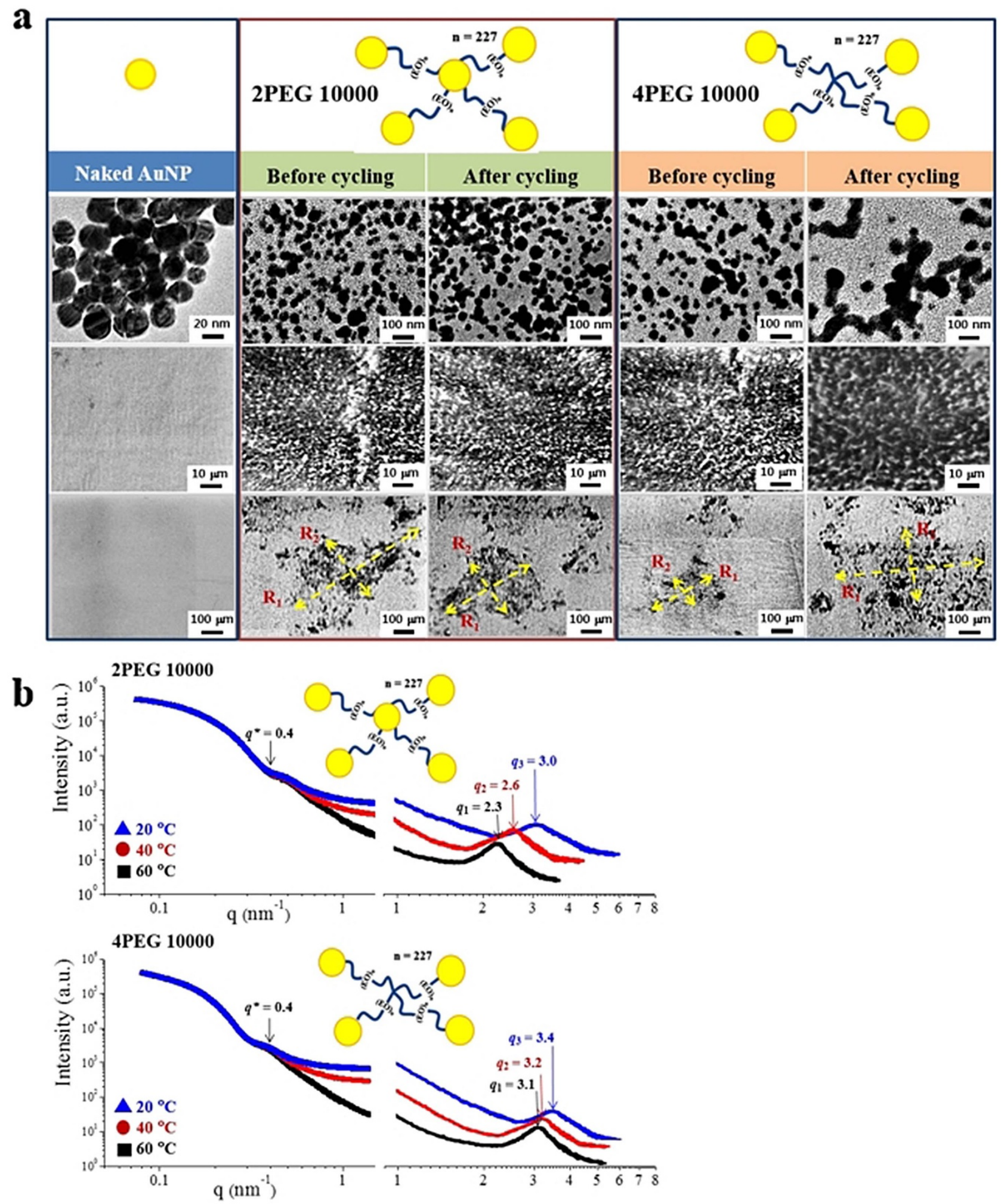

Figure 3 (a) TEM (top line), XNI (middle line) and XMI (bottom line) images before and after the repetition cycling for 2PEG 10000 and 4 PEG 10000 system. (b) SAXS results after the repetition cycling for 2PEG 10000 and 4PEG 10000 systems.

In the third line of Figure 3a, the X-ray micro imaging (XMI) results obtained at the $6 \mathrm{D}$ beam line at PAL provide a larger FOV than XNI (Supporting Information). Spatial resolution is approximately $2 \mu \mathrm{m}$ and FOV is adjusted to $600 \mu \mathrm{m} \times 600 \mu \mathrm{m}$. Au displays high contrast in X-ray images by its high X-ray absorption coefficient ${ }^{27}$. At this imaging condition discrete unit clusters are detected, by which the size of a single cluster can be evaluated. Increased $M_{p}$ of the PEG enhances cluster size with broad interconnection. As the inset arrows in the images illustrate, the average cluster size is evaluated using the arithmetic mean of horizontal $\left(R_{1}\right)$ and vertical $\left(R_{2}\right)$ cluster diameters. Assuming that the cluster is an ellipsoid, the longer length $R_{1}$ is determined on a cluster and then the shorter $R_{2}$ is made at the center point of the $R_{1}$ at a perpendicular angle. Cluster sizes averaged by ten clusters for each case are summarized according to 
the PEG type and the concentration (Figure S1c). In terms of the cycling repetition effect, 2PEG 10000 system does not generate prominent change. However, 4PEG 10000 system exhibits larger cluster in size after the cycling.

Figure $3 \mathrm{~b}$ shows the SAXS results of the 2PEG 10000 and 4PEG 10000 systems after the cycled repetition until around $1000 \mathrm{~min}$. There is no big difference in the peak position of the 2PEG 10000 system before (Figure 1a) and after the cycled repetition (Figure $3 \mathrm{~b}$ ). However, the peaks of the 4PEG 10000 move to the higher $q$ region, indicating structural shrinkage as a results of the cycled repetition. In addition, the size difference induced by the temperature becomes smaller, reflecting temperature insensitivity. These structural changes strongly affect the delayed response to the temperature change in switching function. This SAXS result confirms that the $\xi$ shrinks through the mechanical repetition of responsive cycling, induced by the locally concentrated PEG domains in the nanocomposite. The solubility of PEGs in aqueous condition is a function of the temperature, molecular weight, concentration, additives and so $\mathrm{on}^{35}$. For the designed nanocomposites, the temperature-responsiveness is dominantly affected by the tailored $\mathrm{M}_{\mathrm{p}}$ between the junction points. However, the local concentration of the PEGs in the nanocomposite network is changed by the structural dynamics. Through repeated swelling-deswelling mechanics, the structure of the nanocomposite is rearranged into lower energy state where there is an inherent phase separation of the metallic AuNP regions from the polymeric PEG regions. With induced phase separation, the temperature-responsiveness of the nanocomposite is delayed in a cumulative way (Figure 2d).

Once the AuNP and PEG are chemically linked together, the structure of the nanocomposite is determined even though physical rearrangement occurs further by responsive mechanics. To confirm the time delay in responsiveness is caused by the physical rearrangement of cluster structures, the structure is reorganized by dispersing AuNPs in the nanocomposite homogeneously. The temperatureinsensitive 4PEG 10000 nanocomposites are kept at $4{ }^{\circ} \mathrm{C}$ for a week (with a sonication once a day) and then the AuNPs are redispersed in the nanocomposite modulated by the increased PEG solubility at this low temperature condition. The structural changes are provided in Figure $4 \mathrm{a}$ by the XMI images and the corresponding schematic network illustrations. The aggregation-dispersion of AuNPs is a reversible procedure and this determines temperature-responsiveness of the nanocomposite. The designed nanocomposite structures are dominated by the controlled PEG solubility, in which chemically embedded AuNPs are reorganized reversibly. However, keeping the nanocomposite at high temperature $\left(60^{\circ} \mathrm{C}\right)$ for more than a week does not induce the phase separated state shown in Figure $4 \mathrm{a}$ at the right side. Therefore, it is only by the repeated mechanical processes that modifies the clustering behaviors of chemically linked NP arrangements in the polymer network. Those mechanistic repetitions cannot be reversible simply by temperature-induced solubility control of the polymer.

\section{Discussion}

Inherent properties of responsive materials are induced either by solubility transition or conformation of a macromolecule under certain conditions modulated by external stimuli ${ }^{31}$. The polymer chains in composite materials can be stretched and aligned. This molecular mobility is a typical feature of soft or responsive materials. Typical shape modulation known as rubber deformation is uniaxial based on conventional rubber theory. Length increases in an isotropic way based on $\lambda=L / L_{0}$ (where $L_{0}$ is the initial length and $L$ is the length after stretching), thus width and thickness decrease by the relation of $\lambda^{1 / 2}$. Scale changes in conventional responsive materials are also considered isotropic based on rubber elasticity. However, changes in composite materials deviate from this isotropic deformation due to compositional complexity. Partial responsiveness of the nanocom- posites designed in this study maintains shapes in large-scale while activates valve function in small-scale. This is highly beneficial to be employed for selective mass transport control without physical deformation in macroscale but with effective smart valve performance in nanoscale. The temperature-responsiveness is inherently induced by polymeric domain of PEGs. However through the repeated mechanical cycling of the responsiveness, the polymer domain becomes more concentrated by phase separation where the metallic AuNP domain and polymeric PEG domain are distinctively identified. This induced structural change causes time delay in a cumulative way. With further prolonged mechanistic repetition, the structure of the nanocomposite obtained by XMI changes in a sequence displayed in Figure $4 \mathrm{~b}$. Starting with state [I], more aggregated larger cluster of state [II] and [III] with further time. The nanocomposite reaches the state [IV] where there is a more prominent phase separation between the AuNP cluster region and polymeric film region. The state [III] and [IV] are physically irreversible, thus they do not return to the state [I] by any temperature-controlled aging steps.

Temperature-responsive PEG induces controlled valve functions in the nanocomposite, simultaneously generates phase separation by repeated mechanistic procedures. The nanocomposites having proper nodes composed of the AuNP junctions maintain their structures thus the repeated mechanistic functions. Therefore, properly repositionable NPs chemically embedded in nanocomposites are suggested to be at a metastable state [I] (Figure 4c). Phase-separated state [II] is at the lower energy level. It can be reversibly reached by temperature control due to relatively low activation energy $\left(\mathrm{E}_{\mathrm{a} 1}\right)$ to be easily overcome. However, even though the aggregated sates [III] and [IV] are positioned at the lower energy state, they cannot be reached by physical control by temperature increase due to their relatively high activation energy $\left(\mathrm{E}_{\mathrm{a} 2}\right.$ and $\left.\mathrm{E}_{\mathrm{a} 3}\right)$. To overcome this energy barrier, mechanistic swelling-deswelling repetition performed in this study is suggested. Due to inherent incompatibility between the NPs and PEGs, the phase separated state is most stable even though they are chemically linked. Therefore, in the limit of possible condition, the AuNPs are aggregated while PEGs from filmlike polymeric aggregation. On the images of the state [III] and [IV], phase separated regions are marked by the inset lines demonstrating characteristic wrinkle formation after the repeated mechanistic swelling-deswelling procedures (Figure 4d). The wrinkled lines become bolder with more cycling repetition.

In summary, smart nanocomposites are designed which exhibit high selectivity in mass transport in nanoscale and sharp temperature-responding in a designed time. With structural rearrangements, the nanocomposites display delayed response by repeated mechanical swelling-shrinking procedures. These procedures are similar to those occurring in natural systems where there is a lifetime in life functions. One of the most prominent characteristics of the living systems is a lifetime. A tiredness limits the life function and eventually causes to death at a certain time. Before complete stop (death), the cycling of the live function typically becomes retarded. The designed responsive materials showing characteristic lifetime implies these natural behaviors similarly occurring in biological systems. The designed system in this study is different from the NPpolymer blend system where confinement induces enhancement of NP dispersion ${ }^{36}$. Therefore, the designed system in this study deals with the stimuli-responsiveness rather than thermodynamics dominant for NP phase-separation from polymer domain. Until a certain state, the procedures are reversible but the structural changes and the responding patterns become irreversible with higher deformation in nanoscale structures. To keep the timely-working stimuli-responsiveness, the AuNPs working as stable junction points in the nanocomposite networks are important. This contribution of NP junction points in the stimuli-responsive materials are exclusively reported in this study. The detailed relations between the responding time delays 

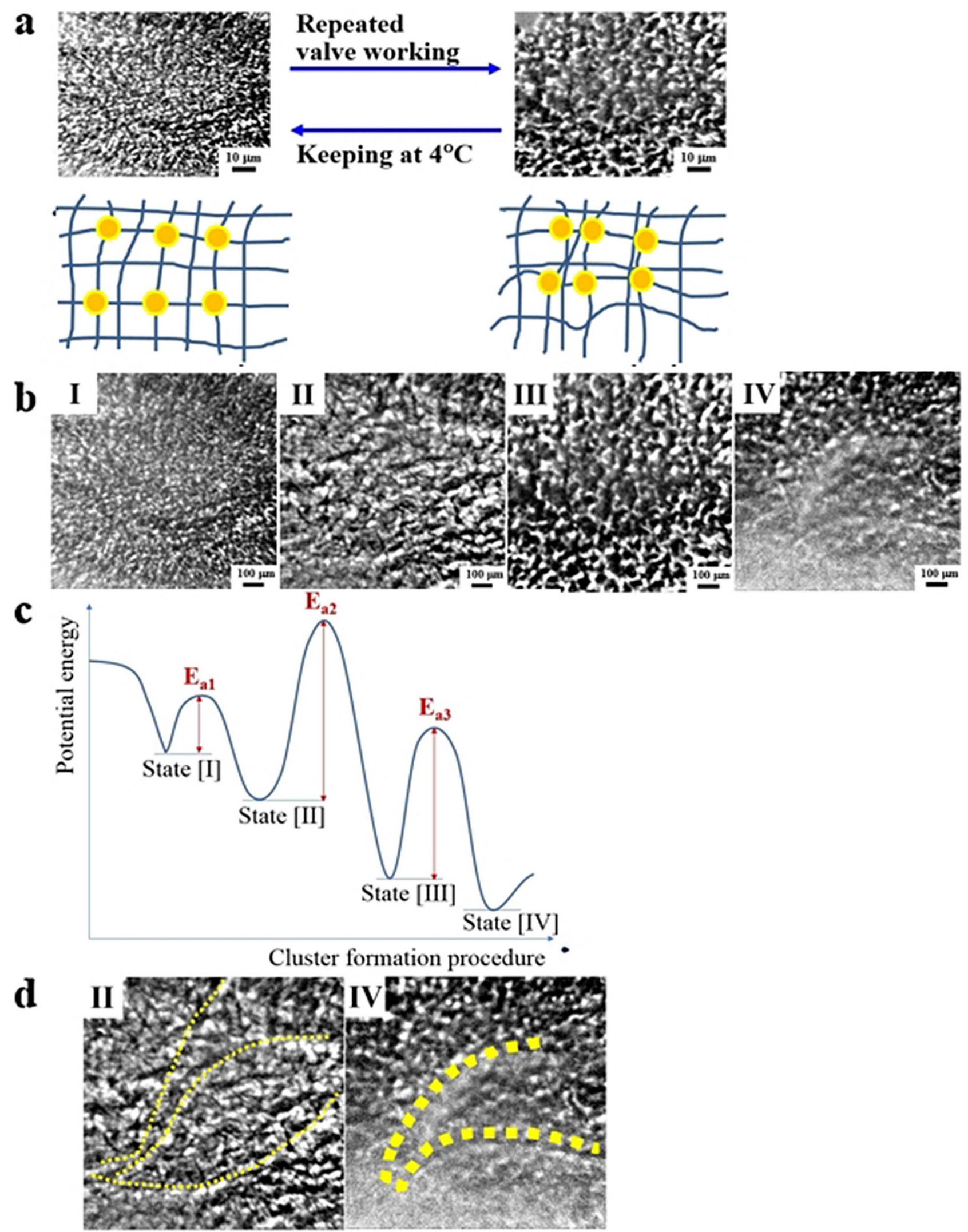

Figure $4 \mid$ (a) XMI image and schematic illustration of the nanocomposite before (left) and after (right) the cyclic repetition. This is a reversible procedure controlled by temperature control. (b) XMI images of nanocomposites obtained with mechanistic repetition from [I] to [IV]. (c) Suggested energy level and activation of the state [I] to [IV] in (b). Due to high activation energy, the state [III] and [IV] is reversible. (d) The wrinkled lines are drawn on the image [III] and [IV]. 
and structural changes are further to be investigated. This study newly illuminates dynamic aspects of the stimuli-responsive materials for broad potential applications.

\section{Methods}

Small-angle X-ray scattering (SAXS). Synchrotron SAXS measurements are performed at $4 \mathrm{C}$ beam line of PAL equipped with a position-sensitive twodimensional (2D) detector. Two energy setting are employed for wavelength modulation: $10 \mathrm{keV}\left(0.0675 \mathrm{~nm}^{-1}\right)$ and $18 \mathrm{KeV}\left(0.1217 \mathrm{~nm}^{-1}\right)$. Samples of $1 \mathrm{~mm}$ thick are used by stacking the five $200 \mu \mathrm{m}$-thick Si wafer of $\mathrm{SiN}_{3}$ sample window. The sample-to-detector distance (SDD) as of $4 \mathrm{~m}$ covers the $q$ range of $0.0679 \mathrm{~nm}^{-1}<q$ $<1.64094 \mathrm{~nm}^{-1}$, where $q=(4 \pi / \lambda) \sin (\theta / 2)$ is the magnitude of the scattering vector and $\theta$ is the scattering angle. The $q$ range is calibrated using polystyrene-blockpoly(ethylene-ran-butylene)-block-polystyrene (SEBS) $\left(q=0.19165 \mathrm{~nm}^{-1}\right)$. On the other hand, the $1 \mathrm{~m} \mathrm{SDD}$ covers the $q$ range of $0.346 \mathrm{~nm}^{-1}<q<7.68039 \mathrm{~nm}^{-1}$. The $q$ range is calibrated using silver behenate $\left(q=1.052 \mathrm{~nm}^{-1}\right)$. A W/B4C double multilayer monochromator are installed to deliver monochromatic X-rays with $6.75 \mathrm{~nm}(18360 \mathrm{keV})$ wavelength and spread of $\Delta \lambda / \lambda=0.01$. The $2 \mathrm{D}$ scattered Xrays are recorded by a CCD camera (Mar CCD, Mar USA, Inc., CCD165). The collected SAXS data are corrected by subtracting the data of background and empty cell scattering. For the system design durability and safety of the system are importantly considered to get reasonable repeatability of the designed nanocomposite. Too high temperature is not aimed not to induce water evaporation of the designed nanocomposite system. Under the given experimental conditions of three ambient temperature condition of 20,40 and $60^{\circ} \mathrm{C}$, we fortunately get noticeable SAXS peak diversification.

Synchrotron X-ray nanoscopy (XN). X-ray imaging is useful for in situ observation of samples. Since vacuum application which is applied in typical image method can distort or further damage AuNP clusters. In this study because of its unique filed-ofview, reasonable resolution and safety for the samples, X-ray imaging is actively employed. Experiments were carried out at the $7 \mathrm{C}$ beam line of the 3rd generation synchrotron radiation source of the PAL. The X-ray source of $10^{11}$ photons $/ \mu \mathrm{m}^{2} / \mathrm{sec}$ consists of undulator with $20 \mathrm{~mm}$ period and 70 poles. The beam size is $100 \mu \mathrm{m} \times$ $100 \mu \mathrm{m}$ at $7 \mathrm{keV}$. The $\mathrm{X}$-ray source is radiated from a $3 \mathrm{GeV}$ bending magnet and then monochromatized using a Ge(111) DCM. For focused images, monochromatic $\mathrm{X}$-ray beam of nominally selected $7 \mathrm{keV}$ is focused on the sample using a condenser zone-plate (CZP, $1 \mathrm{~mm}$ dia. Beryllium refractive compound lenses) with innermost and outermost diameters of $4 \mathrm{~nm}$ and $100 \mathrm{~nm}$, respectively. The primary X-ray image is magnified 50 times using an objective zone plate lens $(140 \mu \mathrm{m}$ innermost and $50 \mathrm{~nm}$ outermost diameter, $\mathrm{W}$ ) and is converted into a visible image on a thin scintillator crystal (Tb:LSO, $20 \mu \mathrm{m}$ thickness). The visible image is further magnified $\times 20$, using an optical microscope, providing a total magnification of $\times 1000$ image on a cooled CCD camera (Princeton Instrument VersArray 1300B cooled CCD) of 1340 pixel $\times 1300$ pixel, which generates an equivalent field of view of $21 \mu \mathrm{m} \times 21 \mu \mathrm{m}$.

X-ray microscopy (XM). Synchrotron X-ray images were captured at $6 \mathrm{D}$ beam line of the PAL. The X-ray source is a bending magnet with critical energy of $8.7 \mathrm{keV}$ at $3 \mathrm{GeV}$ electron energy operation. The white beam is attenuated by polished beryllium (Be) of $0.5 \mathrm{~mm}$ thickness or polished $\mathrm{Si}$ wafer of $1 \mathrm{~mm}$ thickness. The primary X-ray image is converted into a visible image on a thin scintillator crystal CdWO4 of $100 \mu \mathrm{m}$ thickness. X-ray images were captured using a CCD camera (PCO, PCO2000). The field-of-view with a $10 \times$ objective lens in front of the camera was approximately $3.6 \mathrm{~mm} \times 2.4 \mathrm{~mm}$ in physical dimension. The pixel size is about $0.9 \mu \mathrm{m}$

1. Capadona, J. R., Shanmuganathan, K., Tyler, D. J., Rowan, S. J. \& Weder, C. Stimuli-responsive polymer nanocomposites inspired by the sea cucumber dermis. Science 7, 1370-1374 (2008).

2. Ahn, S., Ahn, S. W. \& Song, S. C. Thermothickening modification of the poly(ethylene glycol) and amino acid ester grafted polyphosphazenes by monomethyl end-capped poly(ethylene glycol) addition. Colloid Surf. APhysicochem. Eng. Asp. 333, 82-90 (2009).

3. Ahn, S., Ahn, S. W. \& Song, S. C. Thermosensitive amphiphilic polyphosphazenes and their interaction with ionic surfactants. Colloid Surf. A-Physicochem. Eng. Asp. 330, 184-192 (2008).

4. Skorb, E. V., Sviridov, D. V., Möhwald, H. \& Shchukin, D. G. Light responsive protective coatings. Chem. Commun. 40, 6041-6043 (2009).

5. Psarras, G. C., Parthenios, J. \& Galiotis, C. Adaptive composites incorporating shape memory alloy wires Part I Probing the internal stress and temperature distributions with a laser Raman sensor. J. Mater, Sci. 36, 535-546 (2001).

6. Bhattacharyya, D., Schafer, T., Wickramasinghe, S. R. \& Daunert, S. Responsive Membranes and Materials. Wiley, New York (2013).

7. Carotenuto, G., Martorana, B., Perlo, P. \& Nicolais, L. A universal method for the synthesis of metal and metal sulfide clusters embedded in polymer matrices. J. Mater. Chem. 13, 2927-2930 (2003).

8. Usuki, A., Kojima, M., Okada, A., Fukushima, Y. \& Kamigaito, O. J. Mater. Res. 8, 1179 (1993).

9. Kojma, Y. et al. J. Mater. Res. 8, 1185 (1993).
10. Mirkin, C. A., Letsinger, R. L., Mucic, R. C. \& Storhoff, J. J. A DNA-based method for rationally assembling nanoparticles into macroscopic materials. Nature 382, 607-609 (1996)

11. Elghanian, R., Storhoff, J. J., Mucic, R. C., Letsinger, R. L. \& Mirkin, C. A. Selective colorimetric detection of polynucleotides based on the distance-dependent optical properties of gold nanoparticles. Science 277, 1078-1081 (1997).

12. Storhoff, J. J., Elghanian, R., Mucic, R. C., Mirkin, C. A. \& Letsinger, R. L. One-pot colorimetric differentiation of polynucleotides with single base imperfections using gold nanoparticle probes. J. Am. Chem. Soc. 120, 1959-1964 (1997).

13. Nie, Z., Petukhova, A. \& Kumacheva, E. Properties and emerging applications of self-assembled structures made from inorganic nanoparticles. Nat. Nanotechnol. 5, 15-25 (2010).

14. Iacovella, C. R., Horsch, M. A., Zhang, Z. \& Glotzer, S. C. Phase diagrams of selfassembled mono-tethered nanospheres from molecular simulation and comparison to surfactants. Langmuir 21, 9488-9494 (2005).

15. Iacovella, C. R., Keys, A. S., Horsch, M. A. \& Glotzer, S. C. Icosahedral packing of polymer-tethered nanospheres and stabilization of the gyroid phase. Phys. Rev. E 75, 040801 (2007).

16. Horsch, M. A., Zhang, Z. L. \& Glotzer, S. C. Self-assembly of polymer-tethered nanorods. Phys. Rev. Lett. 95, 056105 (2005).

17. Iacovella, C. R., Horsch, M. A. \& Glotzer, S. C. Local ordering of polymer-tethered nanospheres and nanorods and the stabilization of the double gyroid phase. J. Chem. Phys. 129, 044902 (2008).

18. Mirkin, C. A., Letsinger, R. L., Mucic, R. C. \& Storhoff, J. J. A DNA-based method for rationally assembling nanoparticles into macroscopic materials. Nature $\mathbf{3 8 2}$, 607-609 (1996).

19. Alivisatos, A. P. et al. Organization of 'nanocrystal molecules' using DNA. Nature 382, 609-611 (1996).

20. Tanaka, H., Lovinger, A. J. \& Davis, D. D. Pattern evolution caused by dynamic coupling between wetting and phase separation in binary liquid mixture containing glass particles. Phys. Rev. Lett. 72, 2581 (1994).

21. Lee, B. P., Douglas, J. F. \& Glotzer, S. C. Phys. Rev. E Stat. Phys. Plasmas Fluids Relat. Interdiscip. Topics 60, 5812 (1999).

22. Karim, A., Douglas, J. F., Nisato, G., Liu, D.-W. \& Amis, E. J. Transient Target Patterns in Phase Separating Filled Polymer Blends. Macromolecules 32, 5917 (1999).

23. Balazs, A. C., Ginzburg, V. V., Qiu, F., Peng, G. \& Jasnow, D. Multi-Scale Model for Binary Mixtures Containing Nanoscopic Particles. J. Phys. Chem. B 104, 3411 (2000).

24. Chung, H. -J., Taubert, A., Deshmunkh, R. D. \& Composto, R. J. Mobile nanoparticles and their effect on phase separation dynamics in thin-film polymer blends. Europhys. Lett. 68, 219 (2004).

25. Yurekli, K., Karim, A., Amis, E. J. \& Krishnamoorti, R. Influence of Layered Silicates on the Phase-Separated Morphology of PS-PVME Blends. Macromolecules 36, 7256 (2003).

26. Woodle, M. C., Engbers, C. M. \& Zalipsky, S. New amphipatic polymer-lipid conjugates forming long-circulating reticuloendothelial system-evading liposomes. Bioconjugate Chem. 5, 493-496 (1994).

27. Ahn, S., Jung, S. Y., Lee, J. P., Kim, H. K. \& Lee, S. J. Gold nanoparticle flow sensors designed for dynamic X-ray imaging in biofluids. ACS Nano 4, 3753-3762 (2010).

28. Rabani, E., Reichman, D. R., Geissler, P. L. \& Brus, L. E. Drying-mediated selfassembly of nanoparticles. Nature 426, 271-274 (2003).

29. Martin, C. P., Blunt, M. O. \& Moriarty, P. Nanoparticle networks on silicon: Selforganized or disorganized? Nano. Lett. 4, 2389-2392 (2004).

30. Ahn, S., Ahn, S. W. \& Song, S. C. Polymer structure-dependent ion interaction studied by amphiphilic nonionic poly(organophosphazenes). J. Polym. Sci. Pt. BPolym. Phys. 46, 2022-2034 (2008).

31. Ahn, S., Monge, E. C. \& Song, S. C. Ion and pH effect on the lower critical solution temperature phase behavior in neutral and acidic poly(organophosphazene) counterparts. Langmuir 25, 2407-2418 (2009)

32. Csetneki, I., Filipcsei, G. \& Zrinyi, M. Smart nanocomposite polymer membrane with on/off switching control. Macromolecules 39, 1939-1942 (2006)

33. Schäfer, F. P. (ed.), Dye Lasers 3rd ed.(Springer-Verlag, Berlin, 1990).

34. Dong, D. C. \& Winnik, M. A. The Py scale of solvent polarities. Can. J. Chem. 62, 2560-2565 (1984).

35. Dormidontova, E. \& ten Brinke, G. Phase behavior of hydrogen-bonding polymer-oligomer mixtures. Macromolecules 31, 2649-2660 (1998).

36. Chandran, S., Begam, N., Padmanabhan, V. \& Basu, J. K. Confinement enhances dispersion in nanoparticle-polymer blend films. Nat. Comm. 5, 1-9 (2013).

\section{Acknowledgments}

This work was supported by the National Research Foundation of Korea (NRF) grant funded by the Korea government (MSIP) (No. 2008-0061991). The authors are grateful for the valuable help in the synchrotron X-ray experiments performed at the 6C X-ray micro imaging (XMI), 7C X-ray nano imaging (XNI) and 4C small angle X-ray scattering (SAXS) of the Pohang Accelerator Laboratory (Pohang, South Korea). 


\section{Author contributions}

S.A., S.J.L. developed the concepts. S.A. designed and perform the experiments. S.A. analyzed the results and wrote the paper. All the authors confirm the final version of the manuscript.

\section{Additional information}

Supplementary information accompanies this paper at http://www.nature.com/ scientificreports

Competing financial interests: The authors declare no competing financial interests.
How to cite this article: Ahn, S. \& Lee, S.J. Nanoparticle role on the repeatability of stimuli-responsive nanocomposites. Sci. Rep. 4, 6624; DOI:10.1038/srep06624 (2014).

(c) This work is licensed under a Creative Commons Attribution 4.0 International License. The images or other third party material in this article are included in the article's Creative Commons license, unless indicated otherwise in the credit line; if the material is not included under the Creative Commons license, users will need to obtain permission from the license holder in order to reproduce the material. To view a copy of this license, visit http://creativecommons.org/licenses/by/4.0/ 